\title{
Building an information model in wheel-rail tribosystem control tasks
}

\author{
Vadim Korchagin ${ }^{1 *}$, Andrey Kosmodamianskiy ${ }^{1}$ and Vladimir Vorobiev $^{2}$ \\ ${ }^{1}$ Russian University of Transport (RUT), Dept. of "Traction rolling stock”, 101000 Moscow, Russia \\ ${ }^{2}$ Bryansk State Technical University, Dept. of "Railway rolling stock", 241000 Bryansk, Russia
}

\begin{abstract}
The issues of filling the information model with data on the state of the wheel-rail tribosystem are considered. The "Program for modeling the transverse position of a wheelset in a track" is used, and the main modules of the program are described. The geometrical parameters of the contact of the wheel profile with the rail profile are obtained. The values of the nominal contact of the wheel with the rail for the extreme and intermediate positions of the wheel pair in the rail track are calculated. The displacement of the wheelset in the rail track, its slope, and the width of the contact of the wheel with the rail, the gap between the wheel crest and the inner face of the rail are determined. The obtained data are compared with the results of the experiment.
\end{abstract}

\section{Introduction}

The operation of the tribosystem depends on many factors, including the actual contact area between the bodies [1-6]. The actual contact area is 10-20 times smaller than the nominal area. The magnitude of the transmitted forces, the microgeometry, and macro geometry of the wheel and rail have a significant impact on the size of the contact spot, on friction and wear, especially when working on surfaces. Knowing the value of the instantaneous contact area of the wheel with the rail, it becomes possible to form a control signal for the tribosystem control system. This system is based on an information model that contains information about the parameters of contact between bodies.

The widening of the rail track, the diameter of the wheel, the presence of an elevation of the outer rail, the wear of the upper structure of the track and rolling stock, the geometry of the profile, the amount of elevation of the rail, its sub-slope, the position of the wheel pair in the track form the nominal contact spot. The above factors determine the position of the wheelset in the rail track. A separate assessment of the influence of each factor on the size of the contact spot will allow you to identify the most significant ones and use them further in the construction of an information model in the problems of controlling the wheel-rail tribosystem.

\footnotetext{
* Corresponding author: otvet111333@gmail.com
} 


\section{Materials and Methods}

As part of the solution to the problem of filling the information model, a "Program for modeling the transverse position of a wheelset in a track" was written. The movement of the wheelset in the rail track allows you to determine the points of the greatest elastic penetration on the profile at each position. This program is developed in the Visual LISP environment, the AutoCAD ActiveX Automation Interface is used for data exchange with Visual Basic for Applications, and object-oriented programming technologies were used. The program code consists of 14 modules using the functional programming language AutoLISP, the main structural data is represented by lists.

The program allows you to study the contact of the wheel profile with the rail profile and the size of the gap between them, to determine the shape of the contact spot. The program is based on iterative algorithms. The family of transverse positions of the wheelset is formed by the displacement of the wheelset within the gap between the inner faces of the rail and the ridges, Figure 1.
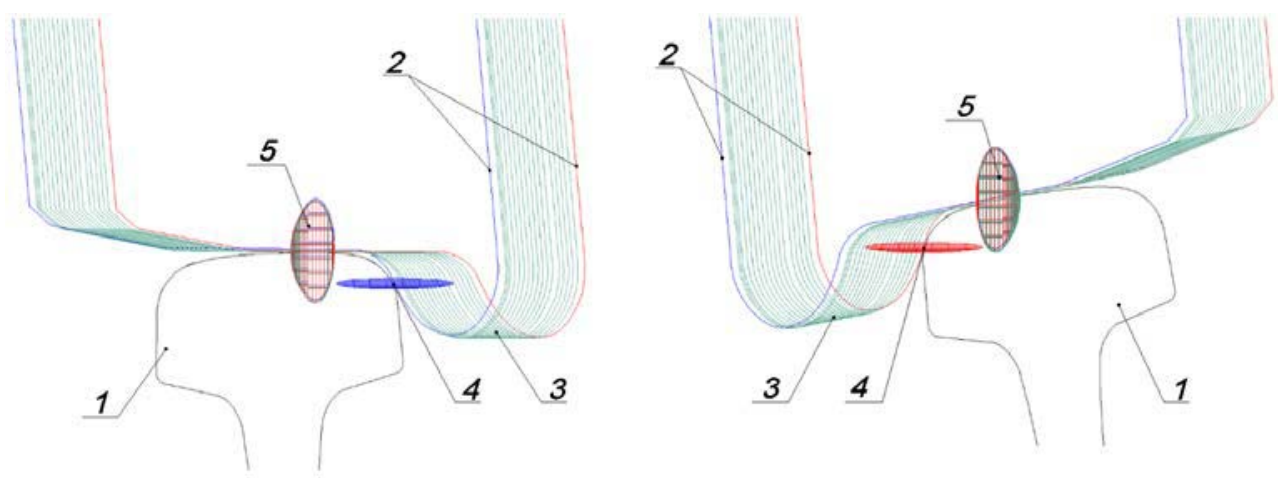

Fig. 1. Family of transverse wheelset positions and nominal rail contact spots.

For the extreme 2 and intermediate 3 positions of the wheelset, the displacement in the rail track 1 , the slope of the wheelset, and the angle of contact of the wheel with the rail is calculated, the shape and area of the contact surface on the ridge 4 and the rolling surface 5 are determined. At each stage of the simulation, the gap between the surfaces of the wheels and the rails is controlled. Compliance with the minimum clearance conditions facilitates the transition to the calculation of the next position of the wheelset in the rail track. After determining the transverse positions of the wheelset in the rail track, the nominal contact area between the wheel and the rail is determined.

The intermediate positions correspond to the case when the ridges of the wheels do not touch the rail, one of the intermediate positions is central. The extreme positions of the wheelset are formed as a two-point contact with the left and right rails. The program input data includes the following parameters: the minimum gap between the wheel and the rail to set the calculation accuracy, the number of intermediate positions of the wheel pair, the wheel diameter around the riding circle, the amount of deformation on the riding surface and the wheel crest, the profile of the wheel pair, the profile of the left and right rails, the presence of rail elevation and track widening. The desired positions of the wheelset in the rail track are determined by iterations of the plane-parallel movement of the wheelset profile relative to the profile of the left and right rails Figure 2. 


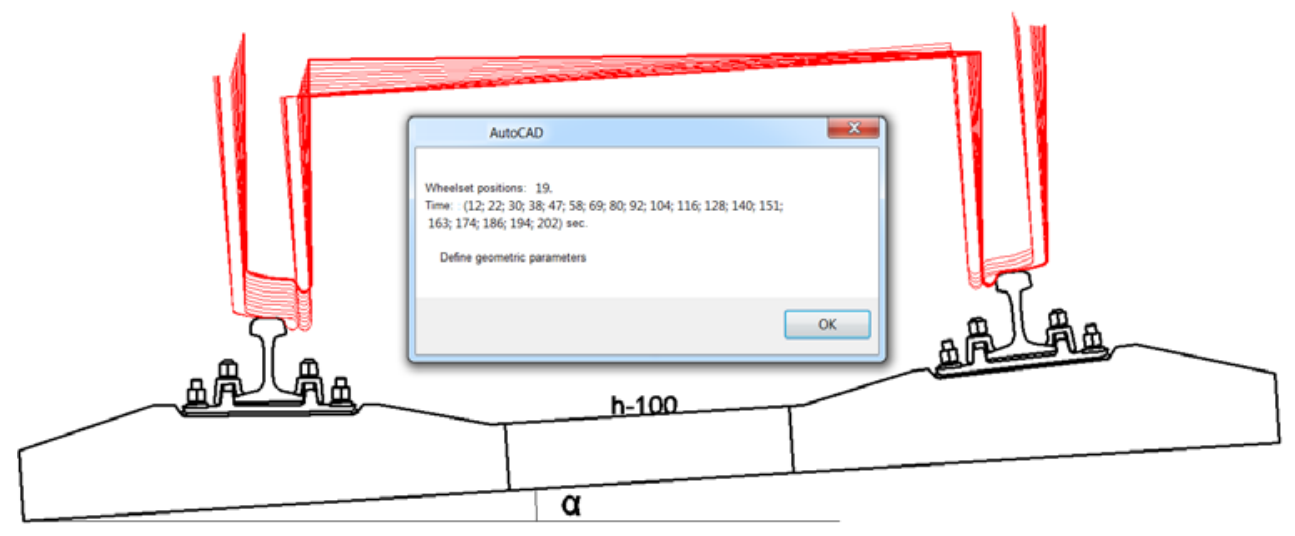

Fig. 2. Iterative determination of the wheelset position in the track.

The point contact, after shifting the profile by the amount of elastic penetration, is transformed into the intersection area. The elongation of the upper boundary of the region in the longitudinal direction and the simultaneous rotation of the lower boundary of the region relative to the axis of the wheelset forms the contact body. The contact body is bounded by the nominal contact surfaces. The calculation time and the number of iterations are displayed in the information messages.

\section{Results and Discussion}

The calculation results and geometric characteristics of the wheel-rail tribosystem are displayed on the screen and saved to a file for further use [7-10]. The geometric parameters of the wheelset positions in the rail track are given in Table 1.

Table 1. Geometric parameters of the wheelset positions in the rail track (wheel diameter-1050 mm; elevation of the outer rail-100 $\mathrm{mm}$ ).

\begin{tabular}{|c|c|c|c|c|c|c|}
\hline \multicolumn{2}{|c|}{ Contact area, $\mathrm{mm}^{2}$} & \multirow{2}{*}{$\begin{array}{c}\text { Wheelset } \\
\text { tilt, }^{\circ}\end{array}$} & \multicolumn{2}{|c|}{ Contact width, $\mathbf{m m}$} & \multicolumn{2}{|c|}{ Clearance, $\mathrm{mm}$} \\
\hline $\begin{array}{c}\text { left } \\
\text { wheel }\end{array}$ & $\begin{array}{l}\text { right } \\
\text { wheel }\end{array}$ & & $\begin{array}{c}\text { left } \\
\text { wheel }\end{array}$ & $\begin{array}{c}\text { right } \\
\text { wheel }\end{array}$ & $\begin{array}{c}\text { left } \\
\text { ridge }\end{array}$ & $\begin{array}{l}\text { right } \\
\text { ridge }\end{array}$ \\
\hline 209 & 218 & 1.65 & 13.2 & 13.9 & 0 & 36 \\
\hline 216 & 242 & 1.67 & 13.6 & 14.7 & -3 & 33 \\
\hline 235 & 245 & 1.71 & 13.4 & 14.7 & -7 & 29 \\
\hline 236 & 244 & 1.74 & 14.1 & 14.6 & -11 & 25 \\
\hline 237 & 244 & 1.76 & 14.1 & 14.5 & -13 & 23 \\
\hline 228 & 237 & 1.77 & 14.3 & 14.5 & -15.5 & 20.5 \\
\hline 239 & 214 & 1.8 & 13.4 & 13.9 & -19 & 17 \\
\hline 240 & 241 & 1.82 & 14.3 & 13.4 & -21 & 15 \\
\hline 241 & 240 & 1.85 & 14.1 & 14 & -25 & 11 \\
\hline 237 & 234 & 1.86 & 14.5 & 14.2 & -27 & 9 \\
\hline 242 & 238 & 1.88 & 14.3 & 14 & -29 & 7 \\
\hline 243 & 210 & 1.9 & 14.5 & 14.1 & -31.5 & 4.5 \\
\hline 217 & 208 & 1.95 & 14.5 & 13.2 & -36 & 0 \\
\hline
\end{tabular}


The simulation results were compared with the nominal contact area of the wheel-rail tribosystem experimentally using a method based on foil printing. Foil was used as an intermediate medium in the wheel-rail tribosystem, and the processing of the foil image made it possible to determine the size of the contact spots between the wheels of the locomotive and the rails. The discrepancy between the calculated and measured areas of the nominal contact did not exceed $10 \%$.

\section{Conclusion}

Since the dimensions of the contact area and the width of the contact spot are practically proportional, one parameter can characterize the other, and by one of these parameters it is possible to detect the conformality of the wheel and rail in the deformed state. The width of the contact spot can be monitored by video recording. The exact values of the nominal contact area of the wheel with the rail are determined by modeling, and the simulation results for worn and new profiles are grouped. After that, information about the size of the contact spot of the wheel - rail tribosystem is transmitted to the system as an information model for solving control problems.

\section{References}

1. C. Foo, B. Omar, A. Jalil, Journal of Physics, A review on recent wheel/rail interface friction management, v. 1049, pp. 17-21 (2018)

2. J. Blanco-Lorenzo, J. Santamaria, E. Vadillo, N. Correa, Tribology International On the influence of conformity on wheel-rail rolling contact mechanics, v. 103, pp. 647667 (2016)

3. D.Y. Antipin, V.I. Vorobyov, V.O. Korchagin, V.V. Kobishchanov, S.G. Shorokhov, Earth and Environmental Science Cep, "Innovations and Prospects of Development of Mining Machinery and Electrical Engineering - Mechanical Engineering", Increasing coupling properties of locomotive by magnetizing contact area of wheel with rail, (2017)

4. D.Ya. Antipin, V.O. Korchagin, M.A. Maslov, Proceedings of the 5th International Conference on Industrial Engineering (ICIE 2019), Determination of Magnetization Efficiency of Wheel-Rail Contact Zone, pp. 669-675 (2020)

5. A. Gerasimenko, X. Liu and Z. Deng, International Conference on Applied Superconductivity and Electromagnetic Devices (ASEMD), Schemes of Turnouts for the Permanent Magnet Guideway of HTS Maglev Vehicles, pp. 1-2 (2020)

6. A. Keropyan, S. Procedia Engineering. Gorbatyuk Impact of Roughness of Interacting Surfaces of the Wheel-Rail Pair on the Coefficient of Friction in their Contact Area, v. 150, pp. 406-410 (2016)

7. W.J. Wang, H.F. Zhang, Q.Y. Liu, M. Zhu, X. Jin, Journal of Engineering Tribology, Investigation on adhesion characteristic of wheel/rail under the magnetic field condition, v. 230, (2015)

8. B. Fenga, AIP Advances, Effects of surface roughness on scratch resistance and stress-strain fields during scratch tests, v. 7, pp. 38-42 (2017)

9. A.S. Kosmodamianskiy, V.O. Korchagin, V.I. Vorobiev, International Conference on Industrial Engineering, Applications and Manufacturing (ICIEAM) Electronic resource, Contact spot for conformal interface of the railroad locomotive wheel with the rail, (2017) 
10.H. Wang, W. Wang, Q. Liu. Journal of Engineering Tribology, Numerical and experimental investigation on adhesion characteristic of wheel/rail under the third body condition, v. 230, pp. 128-132 (2016) 